[Chem. Pharm. Bull.

32( 5$) 2001-2004(1984)]$

\title{
Short-Step Synthesis of Plant Growth-Promoting Brassinosteroids
}

\author{
Suguru TaKatsuto and Nobuo IKeKawa* \\ Department of Chemistry, Tokyo Institute of Technology, \\ Ookayama, Meguro-ku, Tokyo 152, Japan
}

(Received September 26, 1983)

\begin{abstract}
Brassinolide analogues, $(22 R, 23 R, 24 R)-2 \alpha, 3 \alpha, 22,23$-tetrahydroxy- $B$-homo-7-oxa- $5 \alpha$ ergostan-6-one (24-epibrassinolide) (10) and $(22 S, 23 S, 24 R)-2 \alpha, 3 \alpha, 22,23$-tetrahydroxy- $B$-homo7-oxa-5 $\alpha$-ergostan-6-one (9), were synthesized from brassicasterol (3a) in five steps and with $c a$. $20 \%$ overall yield. The key steps are the direct formation of $(22 E, 24 R)-3 \alpha, 5$-cyclo- $5 \alpha$-ergost-22en-6-one (4) from brassicasterol mesylate (3b), the acid-catalyzed rearrangement of 4 to (22E, 24R)-5 $\alpha$-ergosta-2,22-dien-6-one (6), and the Baeyer-Villiger oxidation of the tetrahydroxy$5 \alpha$-ergostan-6-ones 7 and 8 .
\end{abstract}

Keywords_brassinolide; brassinosteroid; plant growth hormone; brassicasterol; BaeyerVilliger oxidation

Brassinolide (1), ${ }^{1)}(22 R, 23 R, 24 S)$ - $2 \alpha, 3 \alpha, 22,23$-tetrahydroxy- $B$-homo-7-oxa-5 $\alpha$-ergostan6-one, and castasterone (2), ${ }^{2)}(22 R, 23 R, 24 S)-2 \alpha, 3 \alpha, 22,23$-tetrahydroxy-5 $\alpha$-ergostan-6-one, are naturally occurring plant growth-promoting steroids. Brassinolide (1) showed a wide variety of biological activities in a number of bioassay systems. ${ }^{3)}$ Among the brassinolide analogues already synthesized, $(22 R, 23 R, 24 R)-2 \alpha, 3 \alpha, 22,23$-tetrahydroxy- $B$-homo-7-oxa- $5 \alpha-$ ergostan-6-one, 24-epibrassinolide (10), and $(22 S, 23 S, 24 R)-2 \alpha, 3 \alpha, 22,23$-tetrahydroxy- $B$ homo-7-oxa-5 $\alpha$-ergostan-6-one (9) are promising candidate compounds for applications in agriculture $^{4)}$ not only because these steroidal lactones were found to be highly active (comparable to brassinolide (1)) in a number of bioassays, ${ }^{5)}$ but also because an efficient synthesis in gram quantities from the commercially available ergosterol, $(22 E, 24 R)$-ergosta5,7,22-trien-3 $\beta$-ol, has been developed by USDA scientists. ${ }^{6)}$ The high biological activity of brassinosteroids 9 and 10 prompted us to report our short-step synthesis of 9 and 10 from brassicasterol, (22E,24R)-ergosta-5,22-dien-3 $\beta$-ol (3a).

In order to introduce $2 \alpha, 3 \alpha$ - and 22,23-vicinal diol functions, (22E,24R)-5 $\alpha$-ergosta-2,22dien-6-one (6) had to be elaborated from ergosterol or brassicasterol via the key intermediate, (22E,24R)-3 $\alpha, 5$-cyclo-5 $\alpha$-ergost-22-en-6-one (4). Thompson et al. $\left.{ }^{6}\right)$ have transformed ergosterol into the 2,22-dien-6-one 6 in eight steps. Anastasia $e$ al $^{7)}$ have obtained brassicasterol (3a) and its $\Delta^{7}$ isomer in a ratio of $3: 2$ from the 1,4-cycloadduct of ergosterol acetate and 4phenyl-1,2,4-triazoline-3,5-dione by reduction with lithium and ethylamine. They transformed brassicasterol (3a) into the 2,22-dien-6-one 6 in five steps. The common transformations to obtain the 3,5-cyclo-6-one 4 are as follows; tosylation, solvolysis to the cyclopropyl-6 $\beta$-ol, and oxidation (and reduction of the C-7 (8) double bond).

Brassicasterol (3a), present in rapeseed oil at $5-19 \%$ of the free sterol fraction ${ }^{8)}$ and now commercially available, is the most suitable starting material for the synthesis of brassinolide analogues 9 and 10. In our case the preparation of the 3,5-cyclo-6-one 4 was achieved by treatment of the brassicasterol mesylate (3b) with sodium acetate ${ }^{9)}$ or potassium acetate in dimethyl sulphoxide at $90-100^{\circ} \mathrm{C}$ in $c a .50 \%$ yield. The by-product $(c a .10 \%)$ was $(22 E, 24 R)-$ ergosta-4,22-dien-3-one (5). The use of triethylamine instead of sodium acetate failed to yield the cyclopropyl ketone 4 . The major product in this case was the dienone 5. Acid-catalyzed 


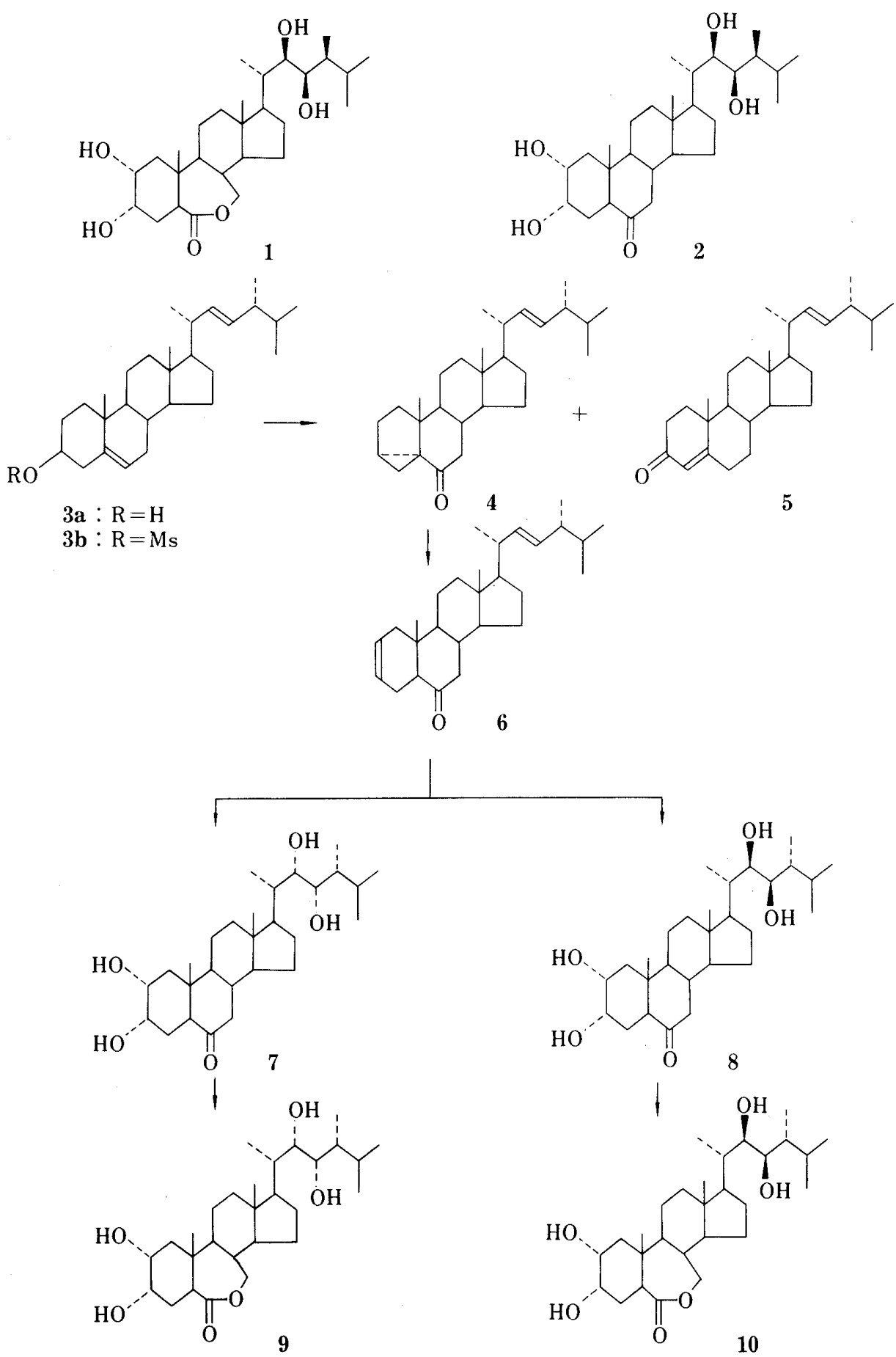

isomerization of the 3,5-cyclo-ketone 4 to $(22 E, 24 R)$-5 $\alpha$-ergosta-2,22-dien-6-one (6) was effected by heating with $p$-toluenesulphonic acid and sulpholane ${ }^{10)}$ in $65 \%$ yield. The 2,22dien-6-one 6 in tert-BuOH-THF- $\mathrm{H}_{2} \mathrm{O}(10: 3: 1)$ was treated with a catalytic amount of osmium tetroxide and 6.5 molar equivalents of $N$-methylmorpholine $N$-oxide ${ }^{11)}$ at room temperature for 3 days to give a separable mixture of the tetraols 7 and 8 . Flash chromatography on silica gel provided the less polar $(22 S, 23 S, 24 R)-2 \alpha, 3 \alpha, 22,23$ tetrahydroxy-5 $\alpha$-ergostan-6-one $\left(7,50 \%\right.$ ), mp $184-185^{\circ} \mathrm{C}$, (lit. ${ }^{7)} \mathrm{mp} 184-185^{\circ} \mathrm{C}$ ) and the more polar $(22 R, 23 R, 24 R)$ - $2 \alpha, 3 \alpha, 22,23$-tetrahydroxy-5 $\alpha$-ergostan-6-one $(\mathbf{8}, 30 \%), \mathrm{mp} 241-$ $242^{\circ} \mathrm{C}$ (lit. ${ }^{7)} \mathrm{mp} 241-242^{\circ} \mathrm{C}$ ). Baeyer-Villiger oxidation ${ }^{12)}$ of 7 and 8 with trifluoroperacetic acid followed by recrystallization of the product provided $(22 S, 23 S, 24 R)-2 \alpha, 3 \alpha, 22,23$ - 
tetrahydroxy- $B$-homo-7-oxa-5 $\alpha$-ergostan-6-one (9), mp 193-195 ${ }^{\circ} \mathrm{C}\left(\right.$ lit. ${ }^{7)} \mathrm{mp} 193-195^{\circ} \mathrm{C}$ ), and $(22 R, 23 R, 24 R)-2 \alpha, 3 \alpha, 22,23$-tetrahydroxy-B-homo-7-oxa-5 $\alpha$-ergostan-6-one (10), $\mathrm{mp}$ $256-257^{\circ} \mathrm{C}$ (lit. ${ }^{7)} \mathrm{mp} 256-257^{\circ} \mathrm{C}$ ), respectively, in $\mathrm{ca} .80 \%$ yield. The overall yield of the five-step synthesis of both 9 and $\mathbf{1 0}$ was $c a .20 \%$.

\section{Experimental}

Melting points were determined with a hot-stage microscope and are uncorrected. Infrared (IR) spectra were recorded on a Hitachi 260-10 spectrometer. Proton nuclear magnetic resonance $\left({ }^{1} \mathrm{H}-\mathrm{NMR}\right)$ spectra were taken with a Hitachi R-24 (60 MHz) spectrometer with tetramethylsilane as an internal standard. Mass spectra (MS) were taken with a Shimadzu LKB-9000S or a Shimadzu GC-MS 6020 mass spectrometer. Column chromatography was done on Kieselgel $60 \mathrm{~F}_{254}$ (70-230 mesh, E. Merck). Analytical thin layer chromatography (TLC) was carried out on precoated Kieselgel $60 \mathrm{~F}_{254}(0.25 \mathrm{~mm}$ thickness, E. Merck). The usual work-up refers to dilution with water, extraction with the organic solvent indicated in parentheses, washing of the extract to neutrality, drying over $\mathrm{MgSO}_{4}$, filtration, and removal of the solvent by evaporation under a vacuum. The following abbreviations are used for ${ }^{1} \mathrm{H}-\mathrm{NMR}$ data; s, singlet; d, doublet; dd, double doublet; $m$, multiplet.

(22E,24R)-3 $\alpha, 5$-Cyclo-5 $\alpha$-ergost-22-en-6-one (4) — Brassicasterol (3a) $(4.1 \mathrm{~g}, 10.3 \mathrm{mmol})$ was treated with methanesulphonyl chloride $(3 \mathrm{ml})$ and pyridine $(20 \mathrm{ml})$ at room temperature for $3 \mathrm{~h}$, then ice-water was added. The whole was extracted with ethyl acetate. The usual work-up gave the mesylate $3 \mathrm{~b}(4.9 \mathrm{~g})$. This was treated with sodium acetate $(5.0 \mathrm{~g}, 60.98 \mathrm{mmol})$ and dimethyl sulphoxide $(160 \mathrm{ml})$ at $90-100^{\circ} \mathrm{C}$ for $5 \mathrm{~h}$. The usual work-up (ether) gave a crude product, which was applied to a column of silica gel $(50 \mathrm{~g})$. Elution with benzene provided $(22 E, 24 R)$-3 $\alpha, 5-$ cyclo- $5 \alpha$-ergost-22-en-6-one (4) $(2.1 \mathrm{~g}, 51 \%), \mathrm{mp} 109-111^{\circ} \mathrm{C}$ (lit. ${ }^{7} \mathrm{mp} 110-111^{\circ} \mathrm{C}$ ) (from aqueous acetone). IR

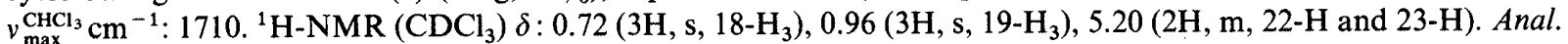
Calcd for $\mathrm{C}_{28} \mathrm{H}_{44} \mathrm{O}$ : C, 84.78; $\mathrm{H}, 11.18$. Found: $\mathrm{C}, 84.66 ; \mathrm{H}, 11.30$.

Further elution with benzene-ethyl acetate $(50: 1)$ gave $(22 E, 24 R)$-ergosta-4,22-dien-3-one (5) $(0.49 \mathrm{~g}, 12 \%), \mathrm{mp}$ $150-151{ }^{\circ} \mathrm{C}$ (from methanol). ${ }^{1} \mathrm{H}-\mathrm{NMR}\left(\mathrm{CDCl}_{3}\right) \delta: 0.74\left(3 \mathrm{H}, \mathrm{s}, 18-\mathrm{H}_{3}\right), 1.15\left(3 \mathrm{H}, \mathrm{s}, 19-\mathrm{H}_{3}\right), 5.20(2 \mathrm{H}, \mathrm{m}, 22-\mathrm{H}$ and 23-H), $6.14(1 \mathrm{H}, \mathrm{s}, 4-\mathrm{H})$. Anal. Calcd for $\mathrm{C}_{28} \mathrm{H}_{44} \mathrm{O}: \mathrm{C}, 84.78 ; \mathrm{H}, 11.18$. Found: C, 84.70; H, 11.21.

(22E,24R)-5 $\alpha$-Ergosta-2,22-dien-6-one (6) - The cyclopropyl ketone $4(2.1 \mathrm{~g}, 5.28 \mathrm{mmol})$ was treated with $p$ toluenesulphonic acid $(100 \mathrm{mg})$ and sulpholane $(16 \mathrm{ml})$ at $160^{\circ} \mathrm{C}$ for $1.5 \mathrm{~h}$. The usual work-up (ether) gave a crude product, which was applied to a column of silica gel $(50 \mathrm{~g})$. Elution with hexane-benzene $(1: 5)$ provided $(22 E, 24 R)$ -

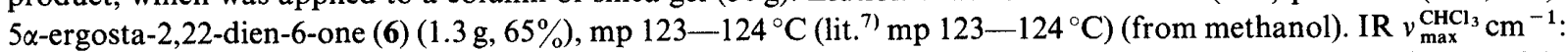
$1705 .{ }^{1} \mathrm{H}-\mathrm{NMR}\left(\mathrm{CDCl}_{3}\right) \delta: 0.68\left(3 \mathrm{H}, \mathrm{s}, 18-\mathrm{H}_{3}\right), 0.70\left(3 \mathrm{H}, \mathrm{s}, 19-\mathrm{H}_{3}\right), 5.20(2 \mathrm{H}, \mathrm{m}, 22-\mathrm{H}$ and $23-\mathrm{H}), 5.60(2 \mathrm{H}, \mathrm{m}, 2-\mathrm{H}$ and 3-H). Anal. Calcd for $\mathrm{C}_{28} \mathrm{H}_{44} \mathrm{O}: \mathrm{C}, 84.78 ; \mathrm{H}, 11.18$. Found: $\mathrm{C}, 84.72 ; \mathrm{H}, 11.27$.

$(22 S, 23 S, 24 R)-2 \alpha, 3 \alpha, 22,23-T e t r a h y d r o x y-5 \alpha$-ergostan-6-one $(7)$ and $(22 R, 23 R, 24 R)-2 \alpha, 3 \alpha, 22,23$-Tetrahydroxy$5 \alpha$-ergostan-6-one (8)-The diene $6(1.3 \mathrm{~g}, 3.43 \mathrm{mmol})$ in tert $-\mathrm{BuOH}-\mathrm{THF}-\mathrm{H}_{2} \mathrm{O}(10: 3: 1,20 \mathrm{ml})$ was treated with osmium tetroxide $(20 \mathrm{mg})$ and $N$-methylmorpholine $N$-oxide $(3.0 \mathrm{~g})$ at room temperature for $3 \mathrm{~d}$, then sat. $\mathrm{NaHSO}_{3}$ solution $(20 \mathrm{ml})$ was added. The mixture was stirred at room temperature for $1 \mathrm{~h}$. The usual work-up (dichloromethane) gave two separable products $(1.5 \mathrm{~g})$, which were purified by flash chromatography. Then $500 \mathrm{mg}$ of the products was applied to a column of silica gel $(3.5 \mathrm{~cm}$ i.d. $\times 15 \mathrm{~cm}$, Kieselgel $60,230-400 \mathrm{mesh}$, E. Merck). Elution with chloroform-methanol $(15: 1)$ provided the less polar $(22 S, 23 S, 24 R)-2 \alpha, 3 \alpha, 22,23$-tetrahydroxy-5 -ergostan-6one (7) $(253 \mathrm{mg}), \mathrm{mp} 184-185^{\circ} \mathrm{C}$ (lit. ${ }^{7)} \mathrm{mp} 184-185^{\circ} \mathrm{C}$ ) (from ethyl acetate). EI-MS $m / z$ : $446\left(\mathrm{M}^{+}-18\right), 394,393$ $\left(\mathrm{M}^{+}-71, \mathrm{C}_{23}-\mathrm{C}_{24}\right.$ fission), $364\left(\mathrm{M}^{+}-101, \mathrm{C}_{22}-\mathrm{C}_{23}\right.$ fission $+\mathrm{H}$, base peak), 363, 345, 327, 287, 263, 245, 175, 173, $155,147,107,101,95,43$. Emitter CI-MS (isobutane) $m / z: 465\left(\mathrm{M}^{+}+1\right.$, base peak), 447, 429. Anal. Calcd for $\mathrm{C}_{28} \mathrm{H}_{48} \mathrm{O}_{5}: \mathrm{C}, 72.41 ; \mathrm{H}, 10.43$. Found: $\mathrm{C}, 72.28 ; \mathrm{H}, 10.50$. Tetraacetate of $7 ;{ }^{1} \mathrm{H}-\mathrm{NMR}\left(\mathrm{CDCl}_{3}\right) \delta: 0.67\left(3 \mathrm{H}, \mathrm{s}, 18-\mathrm{H}_{3}\right)$, $1.93(3 \mathrm{H}, \mathrm{s}$, acetyl), $2.03(9 \mathrm{H}, \mathrm{s}$, three acetyls), 4.50-5.50 $(4 \mathrm{H}, \mathrm{m}, 2-\mathrm{H}, 3-\mathrm{H}, 22-\mathrm{H}$, and $23-\mathrm{H})$.

Further elution with the same solvent gave the more polar $(22 R, 23 R, 24 R)-2 \alpha, 3 \alpha, 22,23$-tetrahydroxy-5 $\alpha$ ergostan-6-one (8) $\left(152 \mathrm{mg}\right.$ ), $\mathrm{mp} 241-242^{\circ} \mathrm{C}$ (lit. ${ }^{7)} \mathrm{mp} 241-242^{\circ} \mathrm{C}$ ) (from ethyl acetate). EI-MS $m / z: 446\left(\mathrm{M}^{+}-18\right)$, $394,393,364,363,345,327,287,263,245,175,173,155,147,107,101,95,43$. Emitter CI-MS (isobutane) $m / z: 465$ $\left(\mathrm{M}^{+}+1\right), 447,429$. Anal. Calcd for $\mathrm{C}_{28} \mathrm{H}_{48} \mathrm{O}_{5}: \mathrm{C}, 72.41 ; \mathrm{H}, 10.43$. Found: $\mathrm{C}, 72.40 ; \mathrm{H}, 10.47$. Tetraacetate of $8 ;{ }^{1} \mathrm{H}-$ NMR $\left(\mathrm{CDCl}_{3}\right) \delta: 0.67\left(3 \mathrm{H}, \mathrm{s}, 18-\mathrm{H}_{3}\right), 1.95(3 \mathrm{H}, \mathrm{s}$, acetyl), $2.00(6 \mathrm{H}, \mathrm{s}$, two acetyls), $2.05(3 \mathrm{H}, \mathrm{s}$, acetyl), 4.65-5.45 $(4 \mathrm{H}, \mathrm{m}, 2-\mathrm{H}, 3-\mathrm{H}, 22-\mathrm{H}$, and $23-\mathrm{H})$. The purification procedure was repeated three times. The total amounts of 7 and 8 were 760 and $457 \mathrm{mg}$ (total yield, $80 \%$ ), respectively.

$(22 R, 23 R, 24 R)-2 \alpha, 3 \alpha, 22,23$-Tetrahydroxy- $B$-homo-7-oxa-5 $\alpha$-ergostan-6-one (10)-Trifluoroperacetic acid was prepared by adding trifluoroacetic anhydride $(6.74 \mathrm{ml})$ to $30 \%$ aqueous $\mathrm{H}_{2} \mathrm{O}_{2}(1.0 \mathrm{~g})$ in dichloromethane $(7.4 \mathrm{ml})$ at $0^{\circ} \mathrm{C}$. Three molar equivalents of the prepared trifluoroperacetic acid solution was added to a solution of the ketone 8 $(400 \mathrm{mg}, 0.86 \mathrm{mmol})$ in dichloromethane $(10 \mathrm{ml})$ at $0^{\circ} \mathrm{C}$. The mixture was stirred at $0^{\circ} \mathrm{C}$ for $3 \mathrm{~h}$, then sat. aqueous $\mathrm{NaHSO}_{3}$ solution $(10 \mathrm{ml})$ was added. The whole was extracted with dichloromethane. The usual work-up and recrystallization from ethyl acetate provided $(22 R, 23 R, 24 R)$ - $2 \alpha, 3 \alpha, 22,23$-tetrahydroxy- $B$-homo-7-oxa-5 $\alpha$-ergostan-6- 
one (10) (343 mg, 83\%), mp 256-258 ${ }^{\circ} \mathrm{C}$ (lit. $\left.{ }^{7)} \mathrm{mp} 256-258^{\circ} \mathrm{C}\right)$. EI-MS $m / z: 465\left(\mathrm{M}^{+}-15\right), 462\left(\mathrm{M}^{+}-18\right), 447,409$, $380\left(\mathrm{M}^{+}-101, \mathrm{C}_{22}-\mathrm{C}_{23}\right.$ fission $+\mathrm{H}$, base peak), 379, 361, 350, 343, 331, 325, 322, 313, 307, 303, 285, 177, 173, 155, 131, 101, 71, 43. Emitter CI-MS (isobutane) $m / z: 481\left(\mathbf{M}^{+}+1\right.$, base peak), 463, 445. Anal. Calcd for $\mathrm{C}_{28} \mathrm{H}_{46} \mathrm{O}_{6}: \mathrm{C}_{\text {, }}$ 70.03; H, 9.85. Found: C, 70.01; H, 9.92. Tetraacetate of $10 ;{ }^{1} \mathrm{H}-\mathrm{NMR}\left(\mathrm{CDCl}_{3}\right) \delta: 0.69\left(3 \mathrm{H}, \mathrm{s}, 18-\mathrm{H}_{3}\right), 1.98(3 \mathrm{H}, \mathrm{s}$, acetyl), $2.02\left(6 \mathrm{H}, \mathrm{s}\right.$, two acetyls), $2.09\left(3 \mathrm{H}, \mathrm{s}\right.$, acetyl), $3.00(1 \mathrm{H}, \mathrm{dd}, J=13$ and $6 \mathrm{~Hz}, 5 \alpha-\mathrm{H}), 4.07\left(2 \mathrm{H}, \mathrm{m}, 7-\mathrm{H}_{2}\right), 4.70-$ $5.50(4 \mathrm{H}, \mathrm{m}, 2-\mathrm{H}, 3-\mathrm{H}, 22-\mathrm{H}$, and $23-\mathrm{H})$. The mother liquor contained some trifluoroacetate of 10 which was recovered by saponification with $5 \% \mathrm{KOH}-\mathrm{MeOH}$ and relactonization with conc. $\mathrm{HCl}$.

$(22 S, 23 S, 24 R)-2 \alpha, 3 \alpha, 22,23-T e t r a h y d r o x y-B$-homo-7-oxa-5 $\alpha$-ergostan-6-one (9)—-The 6-ketone 7 (500 mg, $1.08 \mathrm{mmol}$ ) was oxidized, as described for $\mathbf{1 0}$, to give, after recrystallization from ethyl acetate, $(22 S, 23 S, 24 R)-$ $2 \alpha, 3 \alpha, 22,23$-tetrahydroxy- $B$-homo-7-oxa-5 $\alpha$-ergostan-6-one (9) $\left(415 \mathrm{mg}, 80 \%\right.$ ), mp $193-195{ }^{\circ} \mathrm{C}$ (lit. ${ }^{7)} \mathrm{mp}^{193-}$ $\left.195^{\circ} \mathrm{C}\right)$. EI-MS $m / z: 465\left(\mathrm{M}^{+}-15\right), 462\left(\mathrm{M}^{+}-18\right), 447,409,380,379,361,350,343,331,325,322,313,307,303,285$, 177, 173, 155, 131, 101, 71, 43. Emitter CI-MS (isobutane) $\mathrm{m} / z: 481\left(\mathbf{M}^{+}+1\right.$, base peak), 463, 445. Anal. Calcd for $\mathrm{C}_{28} \mathrm{H}_{48} \mathrm{O}_{6}: \mathrm{C}, 70.03 ; \mathrm{H}, 9.85$. Found: $\mathrm{C}, 69.88 ; \mathrm{H}, 9.87$. Tetraacetate of $9 ;{ }^{1} \mathrm{H}-\mathrm{NMR}\left(\mathrm{CDCl}_{3}\right) \delta: 0.67\left(3 \mathrm{H}, \mathrm{s}, 18-\mathrm{H}_{3}\right)$, $1.93\left(3 \mathrm{H}, \mathrm{s}\right.$, acetyl), $2.03\left(9 \mathrm{H}, \mathrm{s}\right.$, three acetyls), $3.00(1 \mathrm{H}, \mathrm{dd}, J=13$ and $6 \mathrm{~Hz}, 5 \alpha-\mathrm{H}), 4.07\left(2 \mathrm{H}, \mathrm{m}, 7-\mathrm{H}_{2}\right), 4.50-5.50$ $(4 \mathrm{H}, \mathrm{m}, 2-\mathrm{H}, 3-\mathrm{H}, 22-\mathrm{H}$, and 23-H). Some trifluoroacetate of 9 contained in the mother liquor was recovered as 9 by saponification and acidification as described above.

Acknowledgement We thank Tama Biochemical Co., Ltd., for the gift of brassicasterol. We also thank the Ministry of Education, Science and Culture, Japan, for financial support.

\section{References}

1) M. D. Grove, G. F. Spencer, W. K. Rohwedder, N. B. Mandava, J. F. Worley, J. D. Warthen Jr., J. L. FlippenAnderson, and J. C. Cook, Jr., Nature (London), 281, 216 (1979).

2) T. Yokota, M. Arima, and N. Takahashi, Tetrahedron Lett., 23, 1275 (1982).

3) a) J. H. Yopp, N. B. Mandava, and J. M. Sasse, Physiol. Plant., 53, 445 (1981); b) N. B. Mandava, J. M. Sasse, and J. H. Yopp, Physiol. Plant., 53, $453(1981) ;$ c) J. H. Yopp, N. B. Mandava, M. J. Thompson, and J. M. Sasse, 8th Proc. Plant Growth Reg. Soc. Am., 1981, p. 138; d) K. Wada, S. Marumo, N. Ikekawa, M. Morisaki, and K. Mori, Plant and Cell Physiol., 22, 323 (1981); e) L. E. Gregory and N. B. Mandava, Physiol. Plant., 54, 239 (1982).

4) T. H. Maugh II, Science, 212, 33 (1981).

5) a) M. J. Thompson, N. B. Mandava, W. J. Meudt, W. R. Lusby, and D. W. Spaulding, Steroids, 38, 567 (1981); b) M. J. Thompson, W. J. Meudt, N. B. Mandava, S. R. Dutky, W. R. Lusby, and D. W. Spaulding, Steroids, 39, $89(1982)$; c) S. Takatsuto, N. Yazawa, N. Ikekawa, T. Morishita, and H. Abe, Phytochemistry, 22, 1393 (1983); d) S. Takatsuto, N. Yazawa, N. Ikekawa, T. Takematsu, Y. Takeuchi, and M. Koguchi, Phytochemistry, 22, 2437 (1983).

6) M. J. Thompson, N. B. Mandava, J. L. Flippen-Anderson, J. F. Worley, S. R. Dutky, W. E. Robbins, and W. R. Lusby, J. Org. Chem., 44, 5002 (1979).

7) M. Anastasia, P. Ciuffreda, and A. Fiecchi, J. Chem. Soc., Perkin Trans. 1, 1983, 379.

8) T. Itoh, T. Tamura and T. Matsumoto, J. Am. Oil Chem. Soc., 50, 122 (1973).

9) D. Libermann and R. Jacquier, Bull. Soc. Chim. Fr., 1962, 887.

10) a) D. H. R. Barton, P. G. Feakins, J. P. Poyser, and P. G. Sammes, J. Chem. Soc., (C), 1970, 1584; b) K. Mori, Agric. Biol. Chem., 44, 1211 (1980).

11) a) V. VanRheenen, R. C. Kelly, and D. Y. Cha, Tetrahedron Lett., 1976, 1973; b) L. Fieser and M. Fieser, "Steroids," Reinhold, New York, 1959, p. 274.

12) S. Takatsuto and N. Ikekawa, Tetrahedron Lett., 24, 917 (1983). 